children). A 50-mg dose is quite low, but $100 \mathrm{mg}$ does not increase the level or length of focus, for me at least, and can result in nervousness. As no 'high' is achieved, anyone taking too high a dose would soon cut down.

Competitive advantage is not a publichealth issue at all, but a personal ethical and philosophical question. Today I will give my seven-year-old granddaughter a piano lesson, lead her in a chemistry experiment, listen to her sums and encourage her to enter any new words of her vocabulary into her personal dictionary. Am I trying to nurture her towards a 'competitive advantage'? You bet! Charles Eaton

Corrales, New Mexico 87048, USA

\section{Drugging unruly children is a method of social control}

SIR - Sahakian and Morein-Zamir's reference to attention-deficit hyperactivity disorder (ADHD) as heritable and affecting $4-10 \%$ of children worldwide is contentious (Nature 450, 1157-1159; 2007). The claimed incidence of $\mathrm{ADHD}$ varies strikingly over time: less than $0.1 \%$ in the United Kingdom before 1990, and now generally claimed to be between $1 \%$ and $5 \%$. This variation is equally dramatic by country: highest in the United States, followed by Australia and Iceland, but low in Italy, for instance.

The diagnosis is in many cases questionable, and evidence for its heritability is shaky except in highly selected groups. The marked increase in the number of prescriptions of methylphenidate (Ritalin) from 2,000 a year in 1991 to more than 300,000 in the United Kingdom today says more about fashions in the diagnosis and treatment of naughty, inattentive or badly parented children than it does about a genuinely heritable 'disease.'

In the United States, the Federal Drug Administration has called attention to the 'epidemic' of schoolyard Ritalin use. As Sahakian and Morein-Zamir note, there is disturbing evidence of long-term, adverse sequelae associated with the use of such amphetamine-like drugs, especially on young and developing brains.

The assumption behind the cognitiveenhancer debate is that users are essentially making free choices about whether or not to take risks. But children being prescribed Ritalin are being drugged as a method of social control.

That, it seems to me, is a real ethical issue. If we don't recognize the real-world situation in which drugs are bought, prescribed and used, then the ethical debate is vacuous.

Steven Rose

Department of Life Sciences,

The Open University,

Milton Keynes MK7 6AA, UK

\section{Humans have always tried to improve their condition}

SIR - The Commentary 'Professor's little

helper' (Nature 450, 1157-1159; 2007)

entreats us to consider how the non-medical use of cognitive-enhancing drugs such as modafinil and Ritalin might influence society as a whole. They note concerns that a 'better, faster, stronger' mentality might coerce individuals into taking these drugs so that they can give themselves an edge.

Science and technology will continue to generate all sorts of new enhancers, and the quest for enhancement is not necessarily unfair or unethical. We humans are inveterate enhancers, striving to increase our intelligence and to improve our memory and powers of perception.

Consider spectacles: before they became commonplace, those who had good eyesight enjoyed an advantage over those who did not. Later, those who could afford spectacles joined those with naturally good eyesight increasing (or decreasing?) natural unfairness. Enhancing technologies that improve eyesight are now widely available; we do not conclude that they are unethical because they are not globally accessible.

Before the invention of lamps or candles, most people went to bed at dusk; these inventions, and then electricity, enabled social life and work to continue into the night. Night owls can steal a march on their lazier or saner competitors, raising the bar and creating pressure for longer working hours. But such enhancement technologies are not considered unethical.

The same is and will continue to be true of cognitive enhancers. We must press for wider and more equitable access, turning our backs neither on technology nor on improving the human condition.

John Harris, Muireann Quigley

Institute for Science, Ethics, and Innovation,

School of Law, University of Manchester,

Oxford Road, Manchester M13 9PL, UK

\section{Policy must recognize drug impact on different sectors}

SIR - Sahakian and Morein-Zamir encourage us to explore a range of new issues raised by their reflections (Nature 450, 11571159; 2007). In particular, we need to develop legal and social policies to guide the setting of parameters and milestones for integrating new enhancing technologies into healthcare for treatment - and into society for nontherapeutic application.

Policy-making is complex. It becomes even more so when the priorities of different healthcare systems come into play, which are inevitably influenced by the commercial interests of big-business pharmaceutical companies. One-size-fits-all policies will not work because of the range of multicultural factors that also need to be taken into consideration. For example, blanket regulation of cognitive enhancers will not play out evenly where socioeconomic status determines ease of access.

Sahakian and Morein-Zamir call for better drugs. Our call is for next-generation research and translation that is focused on regulatory policies. Those policies should recognize the differential impact of drugs on different segments of society. They should protect people from impulsive quick fixes and against vulnerabilities arising from short-sighted solutions.

Robin Pierce, Judy Illes

National Core for Neuroethics,

Department of Medicine, Division of Neurology,

The University of British Columbia, 2211

Wesbrook Mall, Koerner 5124, Vancouver,

British Columbia V6T 2B5, Canada

\section{Rationality is a better basis for ethics than repugnance}

SIR — Sahakian and Morein-Zamir's Commentary 'Professor's little helper' (Nature 450, 1157-1159; 2007) makes an important contribution to the neuroethics of enhancement, as much for what it doesn't say as for what it does.

Much of the debate over neurocognitive enhancement has been guided by the socalled 'wisdom of repugnance.' We are encouraged to focus on our gut reaction to perfectly healthy individuals drugging themselves (or worse, their healthy children) for the sake of satisfying oversized ambitions. This highlights issues such as the need to earn one's success and self-esteem, and respect for our natural limitations.

Shouldn't we attempt a more rational analysis of the different contexts, methods and motives for neurocognitive enhancement and their likely outcomes, including the likely impact on society and human values?

Sahakian and Morein-Zamir provide a cautious yet open-minded assessment of risks and benefits, without any obeisance to the wisdom of repugnance. They have done us a service in framing the issues in this way.

\section{Martha J. Farah}

Center for Cognitive Neuroscience, University of Pennsylvania, 3720 Walnut Street, Philadelphia, Pennsylvania 19104, USA

\footnotetext{
Contributions to Correspondence may be submitted to correspondence@nature.com. Published contributions are edited. Readers are welcome to contribute to this discussion and many others at http:// network.nature.com.
} 